\title{
Therapie der Nagelpsoriasis
}

\section{Treatment of Nail Psoriasis}

\section{Zusammenfassung}

Psoriatische Nagelveränderungen treten bei der Hälfte der Psoriasispatienten auf. Sie führen neben einer funktionellen Beeinträchtigung zu einer psychischen Belastung des Patienten, da sie im Gegensatz zu den Körperherden nicht ständig verdeckt werden können. Für die Therapie der Nagelpsoriasis stehen lokale und systemische Behandlungskonzepte zur Verfügung, die je nach Schwere der Veränderungen eingesetzt werden können. Trotz verschiedener therapeutischer Optionen ist die Behandlung äußerst schwierig und für Arzt und Patient oft gleichermaßen enttäuschend. Bedingt durch das relativ langsame Nagelwachstum sind Therapieerfolge erst mit großer zeitlicher Verzögerung sichtbar, und nicht selten treten Rezidive auf. Die Behandlung muss daher über mehrere Monate konsequent durchgeführt werden. Es sollen hier die verschiedenen Formen der psoriatischen Nagelveränderungen erläutert werden und ein Überblick über die Therapieoptionen gegeben werden.

\section{Abstract}

Nail psoriasis occurs in as many as $50 \%$ of psoriatic patients. Besides functional limitations the changes in nail-growth lead to cosmetic problems and emotional stress. Treatment of nail psoriasis includes local and systemic regimens. The decision for a special therapeutic agent should depend on the severity of nail psoriasis. Although several drugs have been used for the treatment of nail psoriasis it is still difficult to clear. Because of the relatively slow growth rate of the nail the therapeutic success is seen with a delay of months and relapses are common. The management of nail psoriasis requires a long-term treatment and a compliant patient. This article gives an overview of the different changes in psoriatic nails and shows therapeutic options.

\section{Einleitung}

Etwa 50\% der Patienten mit einer Psoriasis zeigen charakteristische Veränderungen der Nägel. Die Nagelveränderungen können isoliert auftreten, in den meisten Fällen bestehen aber auch typische Psoriasisherde am Körper. Im Rahmen einer Psoriasisarthritis kommt es sogar bei $80 \%$ der Patienten zu einem Befall der Nägel. Der Schweregrad des Nagelbefalls korreliert nicht mit der Ausdehnung der Psoriasis am Integument. Die verschiedenen Formen der Nagelpsoriasis zeigen keine Assoziation mit einem bestimmten Verteilungsmuster der Körperherde. Die Nagelveränderungen im Rahmen einer Psoriasis führen neben einer kos- metischen Beeinträchtigung auch zu einer funktionellen Einschränkung durch die erhöhte Brüchigkeit der Nägel. Zudem sind die Patienten stigmatisiert und beklagen insbesondere, wenn sie beruflich viel Kontakt mit Menschen haben, über eine Einschränkung der Lebensqualität $[1,2]$.

\section{Klinische Formen der Nagelpsoriasis}

Die klinische Manifestationsform der Nagelveränderungen ist abhängig von der betroffenen anatomischen Region des Nagels [3]. Die Veränderungen entstehen durch eine abnorme Zellkine- 
tik und -differenzierung und sind im Allgemeinen reversibel. Prinzipiell können alle Anteile des Nagelapparates betroffen sein: Nagelmatrix, -bett, -platte, Hyponychium, proximaler und lateraler Nagelfalz. Die Nagelmatrix besteht aus dichtgepackten kernhaltigen Zellen. Der proximale Anteil der Matrix bildet die oberflächliche (härtere) Lage der Nagelplatte, der distale Anteil die tiefe (weiche) Lage. Zwischen beiden Lagen besteht eine natürliche Spaltebene. Die Veränderungen an der Nagelplatte im Rahmen einer Psoriasis treten in Abhängigkeit der Lokalisation der Parakeratose im Bereich der Nagelmatrix auf. Am häufigsten ist die proximale Matrix betroffen. Die Nagelveränderungen treten wiederholt, teilweise in regelmäßigen Abständen auf (Tüpfel und Querrillen). Diese Defekte liegen in der oberen Lage der Nagelplatte und können bei starker Ausprägung zu Veränderungen der Nagelfarbe und -textur führen.

Die akute Form der Nagelpsoriasis äußert sich durch eine intensive Entzündung der distalen Phalanx mit einer Deformierung der Nagelplatte bis hin zum Verlust des Nagels.

Die chronische Form ist durch folgende Nagelveränderungen gekennzeichnet.

\section{Tüpfelnägel}

Als Tüpfelnägel oder Grübchen bezeichnet man multiple punktförmige Punzungen der Nagelplatte. Sie entstehen durch eine passagere Hyperproliferation im Bereich der proximalen Nagelmatrix. Dabei kommt es zu einer abnormen Keratinisierung der Zellen mit einer ausgeprägten Parakeratose und Ansammlung von Zellkernresten in der oberflächlichen Lage der Nagelplatte. Beim Herauswachsen des Nagels brechen diese Anteile durch eine verminderte Kohärenz der Zellen untereinander aus der $\mathrm{Na}$ gelplatte heraus und hinterlassen grübchenförmige Defekte (Abb.1). Diese sind meist zufällig verteilt und variieren in Anzahl und Größe. Die Fingernägel sind häufiger betroffen als die Fußnägel.

\section{Leukonychie}

Die Weißverfärbung der Nagelplatte ist auf eine Parakeratose der distalen Nagelmatrix zurückzuführen. Die Nageloberfläche ist dabei intakt. Bei einer Beteiligung der proximalen Nagelmatrix findet man neben der Nagelverfärbung auch Defekte an der Nageloberfläche.

\section{Psoriatische Ölflecken und distale Onycholyse}

Eine Parakeratose im Bereich des Nagelbettes führt zu den psoriasistypischen ovalen, ölfleckartigen Veränderungen, die in Größe und Bestandsdauer variieren. Die gelblich-gräuliche Farbe der Läsionen soll durch eine Akkumulation eines PAS-positiven Glykoproteins unter der Nagelplatte hervorgerufen werden.

Im Bereich des Hyponychiums (Ablösungszone der Nagelplatte vom Nagelbett im Bereich der Fingerbeere) führen parakeratotische Veränderungen zur distalen Onycholyse (Abb.1).

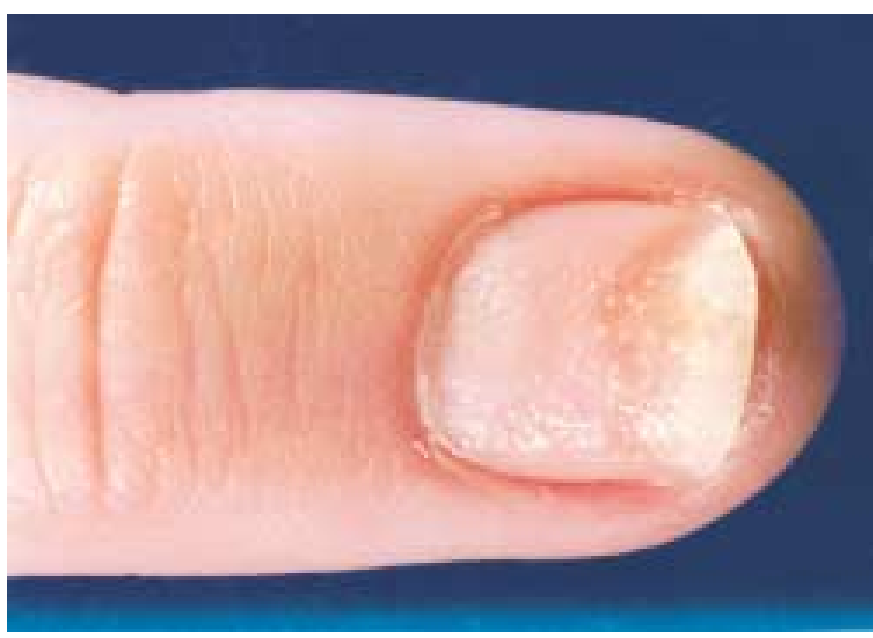

Abb. 1 Typische Nagelpsoriasis mit Tüpfeln und distaler Onlycholyse.

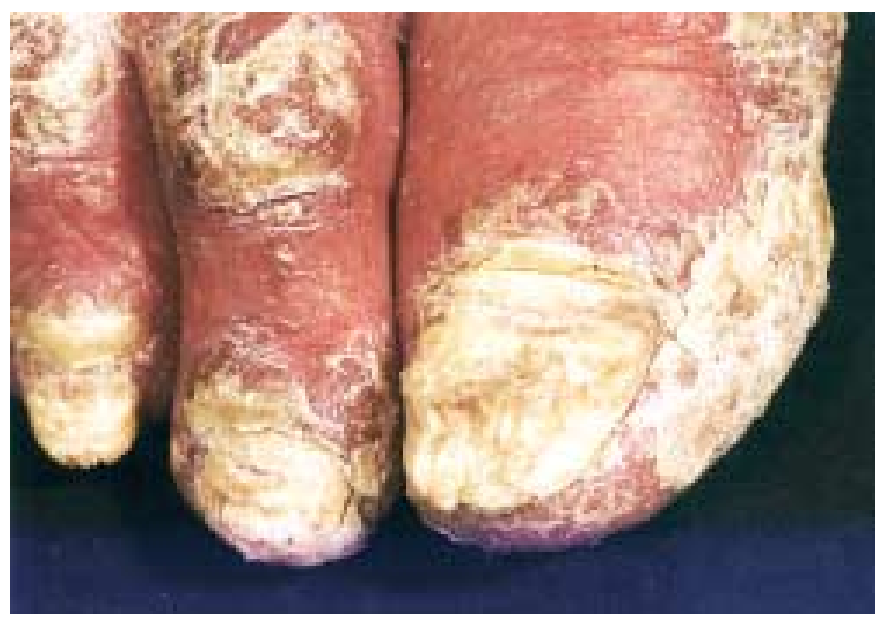

Abb. 2 Befall von proximalem und seitlichem Nagelfalz sowie des gesamten Nagelbettes mit ausgeprägter Onychodystrophie als Maximalvariante einer psoriatischen Onychopathie.

\section{Sulbunguale Hyperkeratose}

Bei dieser häufigen Form der psoriatischen Nagelveränderung finden sich groblamellierte, weißgraue Schuppenmassen unterhalb der Nagelplatte. Sie entstehen durch eine Parakeratose im Bereich des Nagelbettes.

Bei starker Ausprägung kann es zur Abhebung der Nagelplatte und Dystrophie des Nagels kommen (Abb. 2).

\section{Splitterhämorrhagien}

Splitterhämorrhagien sind längsverlaufende Blutaustritte, die als strichförmige, blau-schwarze Verfärbungen vor allem im Bereich des freien Nagelrandes auftreten. Sie entstehen häufig posttraumatisch und betreffen meist die Fingernägel. Auch bei Gesunden findet man diese Nagelveränderung, sie tritt aber bei Psoriasispatienten aufgrund der oberflächlichen Lage der Nagelbettkapillaren häufiger auf. 
Bei dieser Sonderform der Nagelpsoriasis treten sterile Pusteln neben der Nagelplatte auf. Sie führen zu einer Nekrose des Gewebes mit Dystrophie der Nagelplatte. Zusätzlich finden sich Schuppung und Krustenbildung im Bereich des Nagelfalzes. Es handelt sich um ein langsam proximalwärts progredientes Geschehen. Die ausgeprägte entzündliche Aktivität dieser Form der akropustulösen Psoriasis kann zu einem Verlust des Nagels sowie zu einer Vernarbung des Nagelbettes führen. Es können ein oder mehrere Finger und Zehen betroffen sein.

\section{Diagnostik}

In den meisten Fällen kann die Diagnose psoriatischer Nagelveränderungen aufgrund des klinischen Befundes gestellt werden, insbesondere wenn zusätzlich typische Psoriasisherde am Integument bestehen.

Bei Nagelveränderungen an einzelnen Fingern oder Zehen sollte stets eine Pilzinfektion der Nägel durch ein Nativpräparat und eine mykologische Kultur von Nagelmaterial ausgeschlossen werden.

Liegen unklare Nagelveränderungen ohne begleitende diagnostische Hautveränderungen vor, kann es erforderlich sein, eine Biopsie zur Sicherung der Diagnose zu entnehmen. Da alle diagnostischen Phänomene zur histologischen Sicherung einer Nagelpsoriasis im Bereich des Nagelbettes und Hyponychiums nachgewiesen werden können, ist es nicht notwendig die Nagelmatrix in die Probeexzision einzuschließen. So wird eine bleibende Schädigung des Nagelwachstums durch die Probeexzision vermieden.

\section{Therapie}

Die Behandlung der psoriatischen Nagelveränderungen ist nach wie vor ausgesprochen schwierig. Sie ist langwierig, Therapieerfolge sind erst nach einer durch das langsame Nagelwachstum bedingten zeitlichen Verzögerung sichtbar und zufriedenstellende Ergebnisse lassen sich meist nur mit einer systemischen Behandlung erzielen. Die Entscheidung für eine lokale oder systemische Behandlung sollte sich an der Schwere der Veränderungen und der individuellen Beeinträchtigung des Patienten orientieren. Grundsätzlich ist eine gründliche Nagelpflege (Maniküre) bei jeder Form der Therapie hilfreich [4].

Tüpfelnägel und psoriatische Ölflecken bedürfen, da sie nicht zu einer Beeinträchtigung des Nagelwachstums führen, meist keiner Therapie. Sie können bei Bedarf mithilfe von Nagellack zufriedenstellend abgedeckt werden. Alle Formen des psoriatischen Nagelbefalls, die mit einer Ablösung und Zerstörung der Nagelplatte einhergehen, führen neben einer kosmetischen Beeinträchtigung auch zu funktionellen Einschränkungen. In diesen Fällen muss individuell entschieden werden, ob eine systemische Therapie indiziert ist.
Die leichteren Formen der Nagelpsoriasis mit Veränderungen der Nagelsubstanz oder einer distalen Ablösung der Nagelplatte sind einer Lokaltherapie zugänglich. Die längerfristige Anwendung von Kortikoiden sowie Vitamin $\mathrm{D}_{3}$ und dessen Analoga in Form von Lösungen oder Cremes führt gelegentlich zu guten Behandlungsergebnissen. Eine Mischung aus Ecural ${ }^{\circledR}$-Lösung und Propylenglycol zu gleichen Teilen zeigt aufgrund des guten Spreit- und Haftungsvermögens auch unterhalb der Nagelplatte und bei Krümelnägeln eine bessere Wirkung als reine alkoholische Lösungen. Da die Therapie der Nagelpsoriasis langwierig ist und durch das Auftreten von Rezidiven gekennzeichnet ist, muss bei der Verwendung von kortikoidhaltigen Externa immer auch an die Nebenwirkungen (Atrophie der distalen Phalanx) gedacht werden $[5,6]$.

Das Vitamin-D ${ }_{3}$-Analogon Calcipotriol (z.B. Daivonex ${ }^{\circledR} /$ Psorcu$\tan ^{\circledR}$-Lösung) hat eine den topischen Kortikoiden vergleichbare Wirksamkeit bei der Nagelpsoriasis. Der Vorteil liegt in der steroidfreien Behandlungsmöglichkeit und ist so eine sichere Alternative zu den topischen Kortikoiden [7].

Die Lösungen sollten einmal täglich sorgfältig auf der Nagelplatte und am freien Rand des Nagels verrieben werden. Die Behandlung sollte vorzugsweise abends erfolgen, um nicht durch häufiges Händewaschen tagsüber den Wirkstoff wieder zu entfernen. Die Patienten müssen darauf hingewiesen werden, dass die Behandlung über mehrere Monate (mind. zwei) durchgeführt werden muss.

Ein invasiveres Verfahren zur Behandlung der Nagelpsoriasis ist das Unterspritzen befallener Nägel mit Kortikoid-Kristallsuspension. Die Prozedur ist mit einem gewissen Aufwand verbunden, führt aber zu länger andauernden Besserungen psoriatischer Nagelveränderungen als die o.g. Behandlungen. Vor der Unterspritzung der Nägel muss der Finger oder die ganze Hand mittels Leitungsanästhesie betäubt werden. Anschließend wird jeweils medial und lateral unterhalb der Matrix und unterhalb der Nagelplatte ein Depot von 0,05-0,1 ml unverdünnter Kortikoid-Kristallsuspension (z.B. Volon ${ }^{\circledR}$ A 10) mit einer dünnen Kanüle injiziert. Die Behandlung kann nach drei bis sechs Monaten wiederholt werden.

Eine neue Option in der Behandlung von Nagelveränderungen ist das Zalain ${ }^{\circledR}$-Nagelpflaster. Es besteht aus einer PVC-haltigen Trägerfolie und einer Klebematrix. Der physikalische Wirkmechanismus nach dem Prinzip der Okklusion führt zu einer Regulation des Wasserhaushaltes und des Lipidstoffwechsels mit kontrollierten Wachstumsbedingungen. Zusätzlich ist der Nagel mechanisch geschützt. Die Pflaster werden auf die Größe der Nagelplatte zugeschnitten und aufgeklebt. Einmal pro Woche sollten die Pflaster während der mehrmonatigen Behandlung gewechselt werden.

\section{Systemische Therapie}

Bei schweren Formen der Nagelpsoriasis ist nur mit einer systemischen Therapie Aussicht auf Besserung zu erzielen. Die Anwendung von Ciclosporin oder Fumarsäureestern kann bis zur 
vollständigen Erscheinungsfreiheit führen. Bei der Psoriasis-Arthritis stellt Methotrexat das Mittel der ersten Wahl dar. Die bei dieser Form der Psoriasis häufig auftretenden Nagelveränderungen sprechen ebenfalls gut auf diese Behandlung an. Ist aufgrund wenig ausgeprägter kutaner Veränderungen eine systemische Therapie der Psoriasis nicht notwendig oder handelt es sich bei den Nagelveränderungen um die einzigen Symptome einer Psoriasis muss im Einzelfall entschieden werden, ob eine systemische Therapie indiziert ist. Neben der funktionellen Beeinträchtigung durch die erhöhte Brüchigkeit der Nägel und der Stigmatisierung der Patienten, die mit einer Einschränkung der Lebensqualität einhergeht, müssen auch die möglichen Nebenwirkungen einer systemischen Therapie in die Therapieentscheidung einfließen.

\section{Licht- und Röntgenweichstrahltherapie}

Psoriatische Nagelveränderungen sprechen auf eine systemische PUVA-Therapie gut an [10]. Aber auch die lokale PUVA-Therapie der Nägel ist eine gute Behandlungsalternative [11]. Hierfür wird 8-Methoxypsoralen vor der Bestrahlung der Hände mit UVALicht auf die befallenen Nägel und die Nagelfalze aufgetragen.

Die Wirksamkeit von Röntgenstrahlen zur Behandlung der Nagelpsoriasis ist anerkannt. Die Röntgenweichstrahltherapie ist eine Alternative in Fällen, in denen lokale oder systemische Maßnahmen erfolglos waren. Allerdings sollte dieses Therapiekonzept wegen der möglichen Röntgenspätschäden auf Ausnahmefälle begrenzt werden und nur von Ärzten durchgeführt werden, die mit dieser Methode Erfahrung besitzen [12].
Literatur

${ }^{1}$ Zachariae R, Zachariae H, Blomqvist K, Davidsson S, Molin L, Mork C et al. Quality of life in 6497 nordic patients with psoriasis. Br J Dermatol 2001; 146: 1006-1016

${ }^{2}$ Kreuger G, Koo J, Lebwohl M, Menter A, Stern RS, Rolstad T et al. The impact of psoriasis on quality of life: results of a 1998 National Psoriasis Foundation patient-membership survey. Arch Dermatol 2001; 137: $280-284$

${ }^{3}$ Scher RK. The nails in psoriasis. In: Roenigk HH, Maibach HI (eds). Psoriasis. New York, Basel: Dekker, 1985

${ }^{4}$ de Berker D. Management of nail psoriasis. Clin Exp Dermatol 2000; 25: $357-362$

${ }^{5}$ Wolf R, Tur E, Bremer S. Corticosteroid-induced disappearing digit. J Am Acad Dermatol 1990; 23: 755 -756

${ }^{6}$ Requena L, Zamora E, Martin L et al. Acroatrophy secondary to lang standing applications of topical steroids. Arch Dermatol 1990; 126: $1013-1014$

${ }^{7}$ Tosti A, Piraccini BM, Cameli N, Kokely F, Plozzer C, Cannata GE, Benelli C. Calcipotriol ointment in nail psoriasis: a controlled double-blind comparison with betamethasone dipropionate and salicylic acid. $\mathrm{Br} \mathrm{J}$ Dermatol 1998; 139: 655-659

${ }^{8}$ Fredriksson T. Topically applied fluorouracil in the treatment of psoriatic nails. Arch Dermatol 1974; 110: 735-736

${ }^{9}$ Fritz K. Psoriasis of the nail: successful topical treatment with 5-fluorouracil. Z Hautkr 1989; 64: 1083 - 1088

${ }^{10}$ Marx JL, Scher RK. Response of psoriatic nails to oral photochemotherapy. Arch Dermatol 1980; 116: 1023-1024

${ }^{11}$ Handfield-Jones SE, Boyle J, Harman RRM. Local PUVA treatment for nail psoriasis. Br J Dermatol 1987; 116: 280-281

${ }^{12}$ Rassner G. Psoriasis. In: Korting GW (Hrsg). Dermatologie in Praxis und Klinik. 2. Aufl. Stuttgart: Thieme, 1980 